\title{
DESARROLLO DE RASGOS ASOCIADOS A LA AU- TOESTIMA A TRAVÉS DE LA METACOGNICIÓN, EN UNA UNIVERSIDAD MEXICANA
}

\author{
Development of traits related to self-esteem through \\ metacognition in a Mexican university \\ por
}

$\underline{\text { Article record }}$

HTML format

Raúl Augusto Carvallo Castillo ${ }^{a}$

$\underline{\text { Ficha del artículo }}$
$\underline{\text { Formato HTML }}$

\begin{tabular}{l} 
Abstract \\
$\begin{array}{l}\text { We show the effect of the practice of metacognitive } \\
\text { strategies for the change in affective-emotive factors. We } \\
\text { present an empirical study on Mexican university students }\end{array}$ \\
\hline Keywords \\
Self-esteem, metacognition, motivation, university \\
\hline
\end{tabular}

\section{Resumen}

Se pretende poner en evidencia el efecto que pueden ejercer las estrategias metacognitiva para el cambio en factores afectivos-emotivos usando una muestra de estudiantes universitarios mexicanos.

\section{Descriptores}

Autoestima, metacognicion, motivación, universidad

\section{Introducción}

La presente investigación pretende poner en evidencia el efecto que pueden ejercer las estrategias metacognitiva para el cambio en factores afectivos-emotivos. La metacognición se convierte para la persona en "pensar sobre lo que piensa que siente"; -lo cual denomino "Metacognición Afectiva”-, de tal suerte que se desarrolle en ella la conciencia y el control (a través de estrategias) de su emotividadafectividad a partir de su pensamientos sobre su pensamiento de lo que siente

Iniciaré mencionando que en los últimos años se han realizado esfuerzos por dar res- puesta a problemas de aprendizaje con que se enfrentan los alumnos. Actualmente se ha atribuido una mayor importancia a aquellos objetivos relacionados con la enseñanza de procesos y estrategias, así como al desarrollo de programas específicos para enseñar a pensar. En un nivel generalmente independiente se han promovido estudios sobre los aspectos motivacionales referidos al aprendizaje sin llegar a integrarse formalmente en los estudios sobre el desarrollo del pensamiento. Un poco más alejados aún de esta integración educativa han permanecido los estudios sobre el desarrollo del aspecto afectivo o emocional.

\footnotetext{
${ }^{\text {a }}$ Facultad de Psicología (Universidad Veracruzana, México)
} 
Carvallo Castillo, R. A. (2001). Desarrollo de rasgos asociados a la autoestima a través de la metacognición, en una universidad mexicana. RELIEVE, v. 7, n. 2, p. 135-153. http://www.uv.es/RELIEVE/v7n2/RELIEVEv7n2_5.htm

Esto último es precisamente lo que ha motivado el evaluar el uso de estrategias metacognitivas para el desarrollo personal en el ámbito académico y específicamente en el nivel universitario dado que los estudiantes en este momento están en general en su etapa final de preparación académica y en la mayoría de los casos van llevando consigo una serie de preconcepciones equivocadas sobre sí mismos; preconcepciones que fueron aprendiendo a lo largo de su educación preferentemente informal por el peso de algunas delimitaciones culturales y que en la mayoría de los casos afecta a su propio bienestar a pesar de los logros académicos obtenidos.

Es sabido que las personas, desde pequeñas van aprendiendo los aspectos relacionados con el pensamiento formal (a través, o no, de un programa específico) y parecería que los responsables de esta enseñanza consideran casi exclusivamente que el futuro desenvolvimiento social, académico y profesional de los y las niñas, y de los y las jóvenes radica en este pensamiento formal y lógico, tiendendose a desarrollar grandes volúmenes de programas de habilidades de razonamiento y de destrezas (Alonso 1994); y en parte no están equivocados pues resulta innegable la importancia de este desarrollo cognitivo. Sin embargo, por otra parte, se han soslayado (al menos en el campo académico de manera formal y de manera generalizada) la elaboración de programas de desarrollo afectivo-emocional (pudiéndoseles denominar indistintamente de desarrollo del sí mismo, de desarrollo humano, de desarrollo personal, de crecimiento personal, etc. ), programas que por su propia naturaleza complementarían el desarrollo de la persona en un sentido holístico e integrador Resulta difícil ver la exaltación de un programa (de pensamiento formal o de carácter emocional) sin el otro: ¿funcionará con la misma eficacia un programa de operaciones cognitivas básicas si el alumnado "se siente" incapaz de hacerlo? O ¿resultará eficaz un programa de autoestima si el aprendiz no ha desarrollado operaciones cognitivas acordes al programa?. Con respecto a la primera interrogante hay que destacar la importancia que la motivación tiene para el logro (Evans, 1992).

Dado que la motivación esta directamente vinculada con la autoestima y puesto que el trabajo aquí presentado va precisamente en la dirección de este rasgo (autoestima), es preciso puntualizar respecto a este.

La autoestima es un juicio personal de valor, que se expresa en actitudes de la persona hacia sí misma. (Coopersmith, 1991).

Branden $(1994,1996)$ considera a la autoeficacia y a la autodignidad como la esencia de la autoestima. Manifiesta que la experiencia de la autoeficacia genera el sentido de control sobre la propia vida, que asociamos con el bienestar psicológico. Por cuanto a la experiencia de la autodignidad, señala que esta posibilita una actitud afirmativa hacia el derecho de la vida y la felicidad, así como la comodidad al expresar apropiadamente los pensamientos, deseos y necesidades.

Por otra parte, es necesario también explicitar que el presente estudio -como se explicitó en el titulo- se llevará a cabo a través de un programa metacognitivo, el cual en su sentido amplio, requiere un acopio de estrategias que resulten en una dualidad cognición-afectividad que conlleve al crecimiento personal. En este sentido, debe señalarse que la autoestima -en su carácter medular- esta relacionada con los procesos cognitivos, ya que en la medida en que confiemos en la eficacia de nuestra mente (en nuestra capacidad de pensar, aprender comprender) tenderemos a perseverar cuando nos encontramos con desafíos.

Un programa de orientación metacognitiva del desarrollo humano o desarrollo del símismo naturalmente requiere de una explicación específica, ya que generalmente se tiende a asociar el término metacognición en su concepto estructural o procesal en relación a las propias cogniciones, sin establecer su posible asociación en factores emocionales o afectivos,. a menos que estos últimos tengan un papel de variables intervinientes en programas en donde se desea desarrollar alguna habilidad de carácter cognitivo, señalando la importancia o 
Carvallo Castillo, R. A. (2001). Desarrollo de rasgos asociados a la autoestima a través de la metacognición, en una universidad mexicana. RELIEVE, v. 7, n. 2, p. 135-153. http://www.uv.es/RELIEVE/v7n2/RELIEVEv7n2_5.htm

influencia que puede tener en el éxito o fracaso del programa.

Un modelo metacognitivo debe incluir la actividad metacognitiva específica y el objeto propio de esa actividad, el cual es la propia cognición de primer orden. Esta actividad metacognitiva está constituido por dos componentes básicos, la conciencia y el control del proceso.

En el caso presente, en donde se emplean estrategias metacognitivas para el cambio en factores afectivos-emotivos, la metacognición se convierte para la persona en "pensar sobre lo que piensa que siente"; -lo cual denomino "Metacognición afectiva"-., de tal suerte que se desarrolle en ella la conciencia y el control (a través de estrategias) de su emotividadafectividad a partir de su pensamientos sobre su pensamiento de lo que siente

Hilando con lo último expuesto podemos señalar que la significación más relevante de la autoestima es la confianza en sus propios procesos cognitivos

Con base en todo lo anterior, la presente investigación pretende probar la eficacia de un programa de desarrollo humano (de desarrollo del sí mismo, de crecimiento personal, etc.) a través de estrategias metacognitivas, elaborado exprofeso para sujetos de nivel universitario; el cual, dadas sus características bien podría ser utilizado en el nivel previo de estudios (bachillerato).

\section{FORMULACIÓN DE HIPÓTESIS}

Sobre la base del problema planteado, la hipótesis de investigación del presente estudio es:

El desarrollo personal de los alumnos del primer semestre de la Licenciatura en Psicología se incrementará como resultado de la implementación del Programa de Desarrollo Humano a través de Estrategias Metacognitivas.

Las hipótesis estadísticas, nulas y alternas son las siguientes.
Hipótesis de nulidad: Ho: No existe diferencia significativa entre los promedios de los puntajes obtenidos en el inventario (o escala), por los estudiantes a quienes se les aplica el Programa de Desarrollo Humano a través de Estrategias Metacognitivas respecto a aquellos estudiantes a quienes no se les aplica dicho programa.

Hipótesis alterna: Hi: Los promedios de los puntajes obtenidos en el inventario (o escala), son significativamente favorables en aquellos alumnos en que se aplica el Programa de Desarrollo Humano a través de Estrategias Metacognitivas.

\section{Variables}

Las variables involucradas como variable independiente y variable dependiente son respectivamente:

Variable Independiente (VI). La variable a manipularse en la presente investigación es la intervención (o no intervención, grupo control) a través de la aplicación del Programa de Desarrollo Humano a través de Estrategias Metacognitivas (ver anexo 1) en los alumnos del primer semestre de la $\mathrm{Li}$ cenciatura en Psicología de la Universidad Veracruzana.

Variable Dependiente (VD). La variable en la cual se valoran los efectos de la VI es el desarrollo personal alcanzado, el cual es medido a través del puntaje obtenido por los alumnos en los rasgos del CPI (especialmente los asociados a la autoestima) al término de la fase experimental.

\section{MÉTODO}

\section{Sujetos}

En la presente investigación, la población con la cual se trabajó fue la de los estudiantes de recién ingreso a la Facultad de PsicologíaXalapa de la Universidad Veracruzana, en México

Dado que la matrícula anual de ingreso al primer semestre en esta Facultad fue de un máximo de 120 alumnos, estos fueron distribuidos en cuatro grupos existentes asignándo- 
los de manera aleatoria, eligiendo los dos de estudio de la misma forma

\section{Diseño de la investigación}

Para el control de las variables extrañas se empleó el diseño de grupo de control pretestpostest con grupos aleatorizados:

\begin{tabular}{|c|c|c|c|c|}
\multicolumn{5}{|c|}{ Diseño de pretest-postest con grupos aleatorizados } \\
\hline & Grupo & Pretest & $\begin{array}{c}\text { Variable } \\
\text { independiente }\end{array}$ & Postest \\
\hline R) & 1 & $\mathrm{y}_{1}$ & VI & $\mathrm{y}_{2}$ \\
\hline R)) & 2 & $\mathrm{y}_{3}$ & & $\mathrm{y}_{4}$ \\
\hline
\end{tabular}

El análisis estadístico aquí empleado fue tanto el análisis multivariado Wilks' Lambda como el de Pillai-Bartlett Trace.

\section{Instrumentos}

Para la evaluación de los cambios se aplicó el inventario de la Configuración Psicológica Individual (CPI), el cual permite medir los rasgos relacionados con los factores involucrados en el desarrollo afectivo-emocional, dado que se centra en las características de personalidad que tienen una amplia aplicabilidad al comportamiento humano y que están relacionadas con los aspectos favorables, deseables y positivos de la personalidad, tal y como lo menciona su propio autor (Gough H.G., 1990)

A continuación se exponen los rasgos que contempla el CPI:

1.- Do (Dominancia).

2.- Cc (Capacidad de Categoría).

3.- Sd (Sociabilidad).

4.- Ps (Presencia Social).

5.- Aa (Autoaceptación) .

6.- Sb (Sentido de Bienestar).

7.- Re (Responsabilidad).

8.- Sn (Socialización).

9.- Ac (Autocontrol).

10.- To (Tolerancia).

11.- Bi (Buena Impresión).

12.- Co (Comunalidad).

13.- Lc (Logro Conformidad).

14.- Li (Logro Independencia).
15.- Ei (Eficacia Intelectual).

16.- Sp (Sentido Psicológico).

17.- Fx (Flexibilidad).

18.- Fe (Feminidad).

\section{Procedimiento}

La realización de la investigación consistió, en base al diseño, en la aplicación del programa de estrategias metacognitivas para el desarrollo humano (ver anexo 1) en el grupo experimental y la no aplicación en el grupo control. Esto se llevó a cabo en las aulas de la Facultad de Psicología de la Universidad Veracruzana, en Xalapa, Veracruz, México, durante cuatro meses, en dos sesiones por semana, de dos horas y media cada una, dirigidas y supervisadas por el investigador-profesor responsable de los dos grupos, mismo que se encargó de llevar a cabo las evaluaciones pre y pos intervención en ambos grupos.

\section{RESULTADOS}

Para la obtención de los resultados se procedió a la calificación del inventario CPI; la puntuación se realizó de manera directa a través de las plantillas para obtener la calificación natural; posteriormente se convirtió en puntaje estándar.

Para la inferencia estadística se realizó un análisis por medio de las pruebas multivariadas Wilk’s Lambda y Pillai-Bartlett Trace, a través del programa de computo Statistica.

\section{Análisis de los datos del diseño}


Carvallo Castillo, R. A. (2001). Desarrollo de rasgos asociados a la autoestima a través de la metacognición, en una universidad mexicana. RELIEVE, v. 7, n. 2, p. 135-153.

http://www.uv.es/RELIEVE/v7n2/RELIEVEv7n2_5.htm

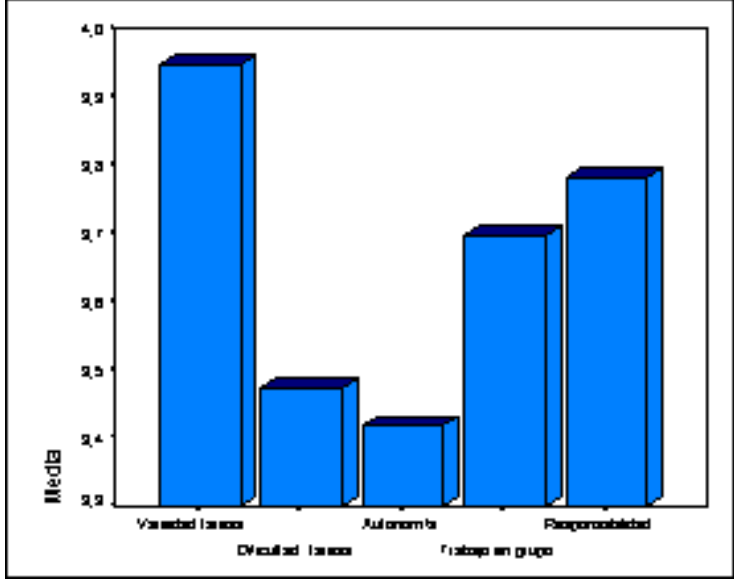

E1

E2

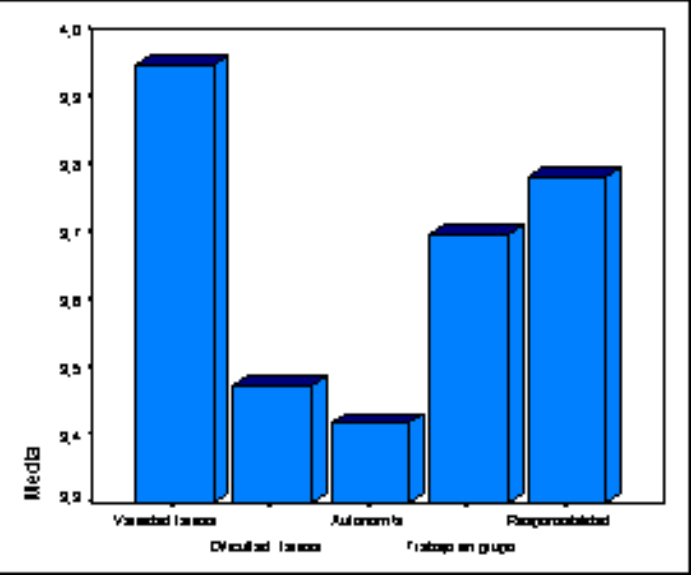

C1
C2
Con base a las medias de los dieciocho rasgos en las cuatro aplicaciones: grupo experimental 1 (E1), grupo experimental 2 (E2), grupo control 1 (C1) y grupo control 2
(C2), se presenta el perfil de medias de los cuatro grupos.

En el perfil de medias pueden visualizarse los cambios existentes entre los cuatro grupos en los diferentes rasgos (fig. 1).

FIGURA 1. Perfil de medias de los cuatro grupos en los dieciocho rasgos.

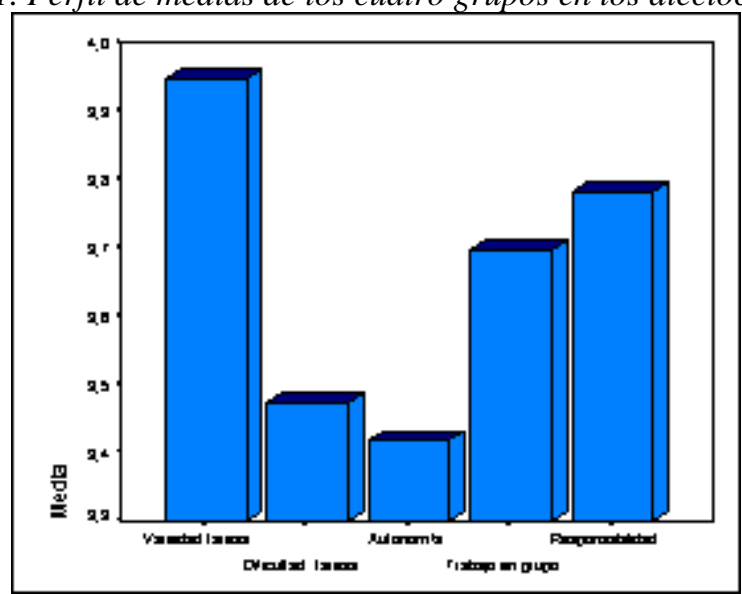

En la gráfica de arriba podemos observar uno a uno los cambios o no cambios aparentes entre los grupos.

En una etapa posterior se procede a un análisis más particular el cual esta centrado en los cambios entre la medición antes-después de cada uno de los rasgos en el grupo experimental, con objeto de apoyo en la visualización de los posibles cambios se presenta una gráfica de cajas de dispersión de los rasgos en la condición antes-después, tanto para el grupo experimental (fig. 2) como para el grupo control (fig. 3). 
Carvallo Castillo, R. A. (2001). Desarrollo de rasgos asociados a la autoestima a través de la metacognición, en una universidad mexicana. RELIEVE, v. 7, n. 2, p. 135-153.

http://www.uv.es/RELIEVE/v7n2/RELIEVEv7n2_5.htm

FIGURA 2. Cajas de dispersión para los rasgos del CPI en el grupo experimental.

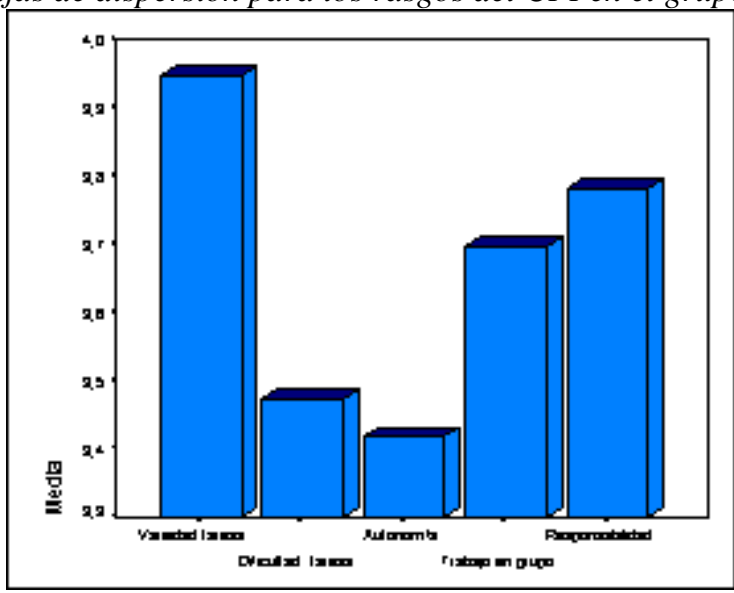

Cont. FIGURA 2. Cajas de dispersión para los rasgos del CPI en el grupo experimental.

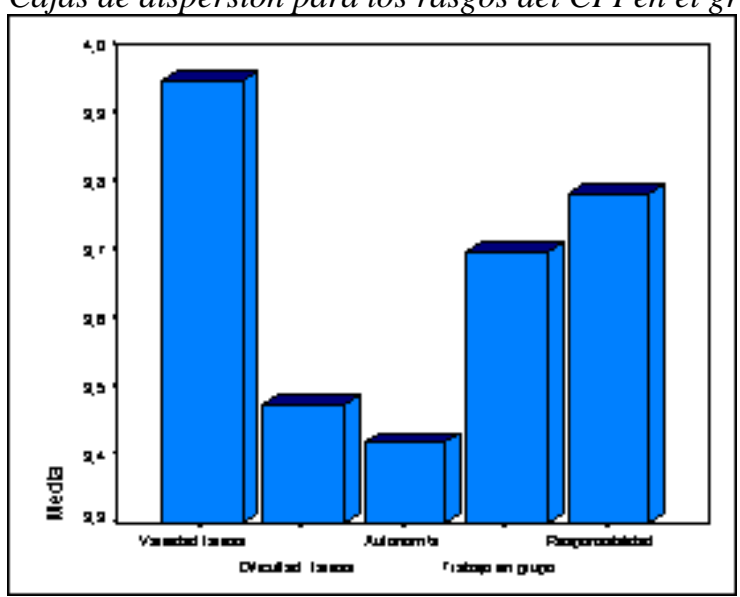

FIGURA 3. Cajas de dispersión para los rasgos del CPI en el grupo control.

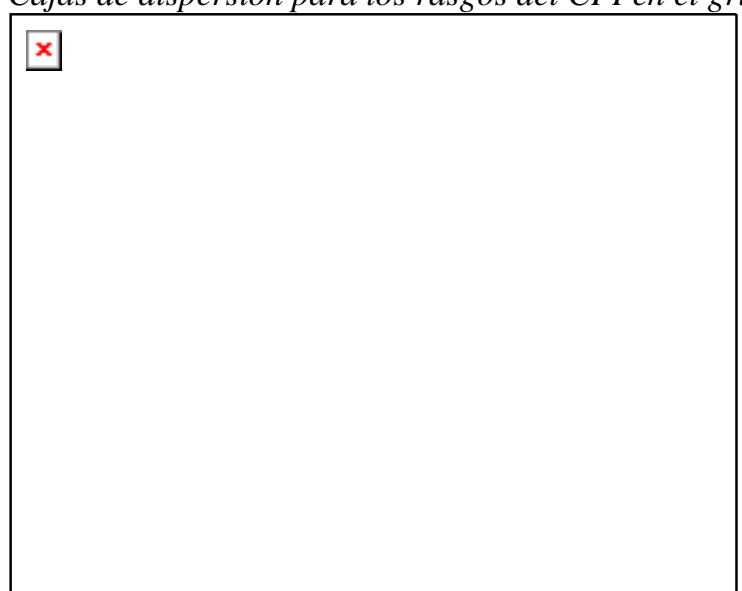


Carvallo Castillo, R. A. (2001). Desarrollo de rasgos asociados a la autoestima a través de la metacognición, en una universidad mexicana. RELIEVE, v. 7, n. 2, p. 135-153.

http://www.uv.es/RELIEVE/v7n2/RELIEVEv7n2_5.htm

Cont. FIGURA 3. Cajas de dispersión para los rasgos del CPI en el grupo control.

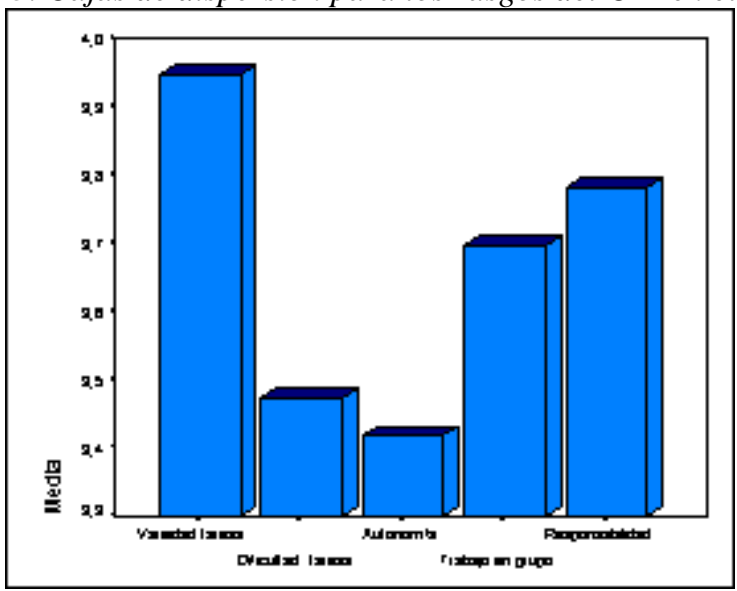

Para la inferencia estadística -como ya se mencionó- se realizó un análisis multivariado, por medio de las pruebas estadísticas Wilk’s Lambda y Pillai-Bartlett Trace, Statistica; las tablas de resultados se irán presentando según se requieran en los análisis.
En el análisis multivariado con las pruebas señaladas, las diferencias entre el total de rasgos para el grupo experimental (antesdespués) resultan ser significativas, $\mathrm{p}<$ 0.005 (tabla 1).

\begin{tabular}{|l|c|c|}
\hline \multicolumn{3}{|c|}{ Cont.TABLA 1 } \\
\hline \multicolumn{1}{|c|}{ Prueba de significancia (anova manova) entre los E1-E2. } \\
\hline Prueba & Valor & Nivel-p \\
Wilks' Lambda & 0.397183 & \\
Rao R Form 2 (18, 35) & 2.951147 & 0.002983 \\
\hline Pillai-Bartlett Trace & 0.602817 & \\
V $(18,35)$ & 2.951147 & 0.002983 \\
\hline
\end{tabular}

\begin{tabular}{|l|c|c|c|c|}
\hline \multicolumn{7}{|c|}{ TABLA 1 } \\
\hline & $\begin{array}{c}\text { Efecto de la media } \\
\text { cuadrada }\end{array}$ & $\begin{array}{c}\text { Error de la media } \\
\text { cuadrada }\end{array}$ & F 1,52 & Nivel -p \\
\hline DO & 0.0000 & 91.2179 & 0.00000 & 1.000000 \\
\hline CC & 220.0185 & 138.6937 & 1.58636 & 0.213471 \\
\hline SD & 411.1296 & 45.9174 & 8.95368 & 0.004228 \\
\hline PS & 140.1667 & 58.9487 & 2.37777 & 0.109137 \\
\hline AA & 253.5 & 23.9858 & 10.56877 & 0.002021 \\
\hline SB & 480.0185 & 53.2365 & 9.01672 & 0.004106 \\
\hline RE & 7.4074 & 64.151 & 0.11547 & 0.735371 \\
\hline SN & 4.1667 & 136.0128 & 0.03063 & 0.861738 \\
\hline AC & 362.963 & 85.2407 & 4.25809 & 0.044072 \\
\hline TO & 6.6852 & 70.7835 & 0.09445 & 0.759827 \\
\hline BI & 88.1667 & 59.1909 & 1.48953 & 0.227795 \\
\hline CO & 200.2963 & 119.7835 & 1.67215 & 0.201686 \\
\hline LC & 121.5 & 124.5712 & 0.97535 & 0.327924 \\
\hline LI & 39.1852 & 83.3262 & 0.47026 & 0.495913 \\
\hline EI & 337.5 & 110.2507 & 3.0612 & 0.086082 \\
\hline SP & 1.5 & 89.2806 & 0.0168 & 0.897368 \\
\hline FX & 140.1667 & 106.114 & 1.32091 & 0.255688 \\
\hline FE & 80.6667 & 32.3661 & 2.49232 & 0.120468 \\
\hline
\end{tabular}


Carvallo Castillo, R. A. (2001). Desarrollo de rasgos asociados a la autoestima a través de la metacognición, en una universidad mexicana. RELIEVE, v. 7, n. 2, p. 135-153.

http://www.uv.es/RELIEVE/v7n2/RELIEVEv7n2_5.htm

Por otra parte el análisis multivariado con las mismas pruebas estadísticas en el grupo control (antes-después) entre los diversos rasgos resulta con diferencias nulas, con una $\mathrm{p}<0.10$ (tabla 2).

\begin{tabular}{|l|c|c|c|c|}
\hline \multicolumn{5}{|c|}{ TABLA 2. } \\
\hline & $\begin{array}{c}\text { Efecto de la media } \\
\text { cuadrada }\end{array}$ & $\begin{array}{c}\text { Error de la } \\
\text { media cuadrada }\end{array}$ & F 1,52 & Nivel -p \\
\hline DO & 21.780 & 92.6000 & 0.235205 & 0.629897 \\
\hline CC & 32.000 & 153.9583 & 0.207848 & 0.650515 \\
\hline SD & 79.380 & 79.2967 & 1.001051 & 0.322074 \\
\hline PS & 188.180 & 173.7300 & 1.083175 & 0.303202 \\
\hline AA & 106.580 & 104.9667 & 1.015370 & 0.318673 \\
\hline SB & 106.580 & 204.8050 & 0.520397 & 0.474172 \\
\hline RE & 184.320 & 64.2367 & 2.869389 & 0.096760 \\
\hline SN & 343.220 & 181.9367 & 1.886481 & 0.175981 \\
\hline AC & 0.320 & 81.8683 & 0.003909 & 0.950409 \\
\hline TO & 297.680 & 95.2300 & 3.125906 & 0.083413 \\
\hline BI & 48.020 & 77.5583 & 0.619147 & 0.435232 \\
\hline CO & 1200.50 & 199.9350 & 6.004451 & 0.017966 \\
\hline LC & 98.000 & 146.4567 & 0.669140 & 0.417394 \\
\hline LI & 0.320 & 78.1833 & 0.004093 & 0.949255 \\
\hline EI & 386.420 & 156.0450 & 2.476337 & 0.122140 \\
\hline SP & 8.820 & 68.4617 & 0.128831 & 0.721221 \\
\hline FX & 188.180 & 78.7983 & 2.388122 & 0.128829 \\
\hline FE & 169.280 & 116.1133 & 1.457886 & 0.233183 \\
\hline
\end{tabular}

Cont. TABLA 2.

Prueba de significancia ( anova, manova) entre los grupos C1-C2.

\begin{tabular}{|l|l|l|}
\hline \multicolumn{3}{|c|}{ Prueba de significancia ( anova, manova) entre los grupos C1-C2. } \\
\hline Prueba & Valor & Nivel-P \\
\hline Wilks' Lambda & 0.643179 & \\
Rao R Form 2 (18, 31) & 0.955451 & 0.527942 \\
\hline Pillai-Bartlett Trace & 0.356821 & \\
V (18,31) & 0.955451 & 0.527942 \\
\hline
\end{tabular}

Pasamos ahora a realizar un análisis específico de los cambios ocurridos o no ocurridos en cada uno de los rasgos.

Con respecto al rasgo Dominancia (DO) se observa como la media de las cuatro condiciones de aplicación se aglutinan alrededor de las medias 45 y 46 (fig. 1), refiriendo esto una nula diferencia entre las cuatro medias. Así mismo observamos esta nula diferencia entre las cajas de dispersión tanto en el grupo experimental (fig. 2) como en el grupo control (fig. 3). En cuanto a la significancia estadística del grupo experimental (E1-E2) y del grupo control (C1-C2) encontramos en ambos una $\mathrm{p}>0.10$, concluyendo que tanto en el grupo experimental como en el grupo control no existen diferencias significativas para este rasgo (DO) en la medición antesdespués (tablas 1 y 2 ).

En cuanto al rasgo Capacidad de Categoría (CC) observamos en el perfil de medias un ligero cambio en el grupo experimental entre la medición antes-después (fig. 1); el mismo efecto puede verse en las cajas de dispersión (fig. 2). 
Carvallo Castillo, R. A. (2001). Desarrollo de rasgos asociados a la autoestima a través de la metacognición, en una universidad mexicana. RELIEVE, v. 7, n. 2, p. 135-153.

http://www.uv.es/RELIEVE/v7n2/RELIEVEv7n2_5.htm

Sin embargo estadísticamente no resulta significativo este cambio, ya que tiene una $\mathrm{p}$ $>0.10$ (tabla 1), en el grupo control no se observa ningún cambio importante como puede verificarse en el perfil de medias (fig. 1) y en las cajas de dispersión (fig. 2); su probabilidad también resulta ser mayor a $\mathrm{p}>$ 0.10 (tabla 2).

En el rasgo Sociabilidad (SD) si encontramos una clara diferencia entre la media obtenida en el pretest y la media obtenida en el postest, aspecto que no se presenta en el grupo control (fig. 1). En las cajas de dispersión también es clara la separación de la distribución de la variabilidad para el grupo experimental en la medición antes-después (fig. 2) situación que no ocurre en el grupo control (fig. 3)

El cambio observado en el grupo experimental para este rasgo tuvo una $\mathrm{p}<0.01$ (tabla 1); en el grupo control no hubo ningún cambio significativo ya que obtuvo una $\mathrm{p}>$ 0.10 (tabla 2).

La Presencia Social (PS) resulta con un cambio visible en las medias de las mediciones antes-después del grupo experimental (fig. 1), pero no lo suficiente para ser significativo ya que aunque tiene una probabilidad extremadamente cercana a 0.10 , esta resulta ser ligeramente mayor (0.1091) (tabla 1). El grupo control presenta también cambios insignificantes con una $\mathrm{p}>0.10$ (tabla 2).

Las cajas de dispersión permiten ver también como las dispersiones tienden a separarse mas en el grupo experimental (fig. 2) que en el grupo control (fig. 3).

Un rasgo importante en esta investigación es el de Autoaceptación (AA), el cual resulta con cambios significativos en el grupo experimental con una $\mathrm{p}=0.002$ (tabla 1 ), mas no en el grupo control, ya que sus cambios tienen una $\mathrm{p}^{>} 0.10$ (tabla 2). La diferencia de ambos grupos puede verse en el perfil de medias en donde se observan claramente los cambios entre los puntajes medios de E1 y E2 (fig. 1); de igual forma en las cajas de dispersión del grupo experimental se puede ver la marcada separación de ambas distribuciones de variabilidad (fig. 2), cosa que no ocurre en el grupo control (fig. 3).

Otro rasgo fundamental en la investigación es el Sentido de Bienestar (SB) el cual obtuvo cambios significativos en el grupo experimental con una $\mathrm{p}=0.004$ (tabla 1 ). El grupo control no observó cambios significativos al obtener una $\mathrm{p}>0.10$ (tabla 2). Los cambios manifestados pueden verse en el perfil de medias en donde se observa como el cambio en el grupo experimental supera al cambio presentado en el grupo control (fig. 1). De igual manera las cajas de dispersión en el grupo experimental dan cuenta de la separación significativa entre ambas distribuciones de variabilidad (fig. 2), en tanto que en el grupo control de hecho estas distribuciones se sobreponen una a otra (fig. 3), denotando un ligero cambio no significativo.

La Responsabilidad (RE) es un rasgo que no representa cambios visibles entre las medias del grupo experimental en las situaciones antes-después, pero que presenta un pequeño cambio en el grupo control (fig. 1) aunque como en el primer caso, resulta no significativo dado que posee una $\mathrm{p}>0.05$ (tablas 1 y 2). Las cajas de dispersión (fig. 2) para el grupo experimental permiten ver la sobreposición de los resultados medios y de dispersión en las condiciones antes después.

Las cajas de dispersión para el grupo control (fig.3) muestran este ligero cambio no significativo.

La Socialización (SN) es un rasgo que tampoco consiguió diferencias significativas ni en el grupo experimental ni en el control ya que en ambos casos obtuvo una $\mathrm{p}>0.10$ (tablas 1 y 2) Por lo anterior hay que interpretar con reserva el cambio que se observa en el postest del grupo control en el perfil de medias (fig. 1).

Con respecto a las cajas de dispersión, las del grupo experimental muestran claramente la sobreposición entre la medición antes y la 
Carvallo Castillo, R. A. (2001). Desarrollo de rasgos asociados a la autoestima a través de la metacognición, en una universidad mexicana. RELIEVE, v. 7, n. 2, p. 135-153.

http://www.uv.es/RELIEVE/v7n2/RELIEVEv7n2_5.htm

medición después (fig. 2); en tanto que para el grupo control (fig 3) muestran un desplazamiento de la "condición después" respecto de la "condición antes", aunque como ya se indicó este cambio no es significativo.

Referente al rasgo denominado Autocontrol (AC) puede observarse en el perfil de medias (fig. 1) como, en tanto que el grupo control de hecho no cambia, en el grupo experimental se aprecia un cambio sustancial.

En relación a las cajas de dispersión correspondientes a este rasgo se observa también un desplazamiento de la condición postest respecto de la condición pretest cercana al límite de la primera desviación (fig. 2), en tanto que en el grupo control las condiciones pretest-postest permanecen casi superpuestas (fig. 3).

El nivel de significancia obtenido en el cambio del grupo experimental fue de una $\mathrm{p}<$ 0.05 (tabla 1) en tanto que para el grupo control se obtuvo una $\mathrm{p}>0.10$ (tabla 2 ).

El rasgo Tolerancia (TO) no presenta cambios visibles entre las medias del grupo experimental en las condiciones pretest-postest, y aunque se observa un ligero cambio entre las medias del grupo control (fig. 1), este resulta no significativo dado que tiene una $\mathrm{p}>0.05$ en ambos casos (tablas 1 y 2) .Las cajas de dispersión para el grupo experimental (cont fig. 2) permiten ver la sobreposición de los resultados medios y de dispersión en las condiciones pretest-postest. Las cajas de dispersión para el grupo control (cont fig. 3) muestran este ligero cambio no significativo.

En cuanto al rasgo Buena Impresión (BI), podemos ver que este muestra en el perfil de medias, puntajes muy cercanos entre sí tanto para el grupo control como para el experimental (fig. 1). Aunque el grupo experimental muestra en las cajas de dispersión un cambio ligero en la media (cont fig. 2) este no resulta suficiente y la distribución del postest se mantiene casi dentro del area de la distribución del pretest. Los cambios en este rasgo, tanto para el grupo experimental como para el grupo control resultan no significativos dado que poseen una $\mathrm{p}>0.10$ (tablas $1 \mathrm{y}$ 2).

El rasgo Comunalidad (CO) resultó con cambios no significativos para el grupo experimental quien tuvo una $\mathrm{p}>0.10$ (tabla 1 ), y con cambios significativos en el grupo control con un $\mathrm{p}<0.05$ (tabla 2); ;lo anterior se corrobora en las correspondientes cajas de dispersión.(cont fig.2).

En las cajas de dispersión del grupo control (cont fig. 3) puede observarse el desplazamiento de los puntajes hacia arriba en la "condición después", quedando incluso su media, fuera de los límites de la segunda desviación de la distribución de los puntajes en la "condición antes". El perfil de medias (fig. 1) muestra el escaso cambio en el grupo experimental y la gran diferencia entre las medias del grupo control en la condición antes-después.

El rasgo Logro Conformidad (LC) alcanzó un pequeño cambio muy similar en ambos grupos, experimental y control, como puede observarse en el perfil de medias (fig. 1) y en las cajas de dispersión correspondientes (cont figs. 2 y 3), sin embargo el traslapamiento de ambas distribuciones (pretest-postest) resulta evidente en esta gráfica de cajas para ambos grupos. El cambio en ambos grupos resulta no significativo con una $\mathrm{p}>0.10$ (tablas 1 y 2 ).

El Logro Independencia (LI) es otro rasgo que al igual que en el anterior resultó con cambios no significativos con una $\mathrm{p}>0.10$ para ambos grupos (tablas 1 y 2).

Las cajas de dispersión (cont. fig. 2) permiten observar esta sobreposición en las distribuciones de las condiciones pretest-postest del grupo experimental. El traslapamiento en la distribución del grupo control se observa contundente en las correspondientes cajas de dispersión (cont. fig. 3). El perfil de medias (fig. 1) da cuenta del grado de aglutinamiento de las cuatro mediciones. 
Carvallo Castillo, R. A. (2001). Desarrollo de rasgos asociados a la autoestima a través de la metacognición, en una universidad mexicana. RELIEVE, v. 7, n. 2, p. 135-153.

http://www.uv.es/RELIEVE/v7n2/RELIEVEv7n2_5.htm

El rasgo Eficiencia Intelectual (EI), resultó con cambios no significativos para el grupo control con una p> 0.10 (tabla 2), el grupo experimental por su parte no resultó significativo a un nivel de $\mathrm{p}<0.05$ pero si a un nivel de $\mathrm{p}<0.10$ (tabla 1 ). El cambio observado en el grupo experimental, aunque no contundente pero significativo, es conveniente tenerlo en consideración.

En las cajas de dispersión para el grupo experimental (cont. fig. 2) puede observarse el desplazamiento de la distribución, en donde la media de postest, se encuentra casi en los limites de la segunda desviación del pretest. En las cajas de dispersión para el grupo control -en el que hubo un cambio no significativo- aunque se percibe un cierto desplazamiento, se observa como ambas cajas parecen tocarse a nivel de la primera desviación (cont fig. 3).

El perfil de medias (fig. 1) nos muestra los cambios en las medias de ambos grupos (antes-después), aunque sólo resultó significativo el grupo experimental.

Con relación al rasgo Sentido Psicológico (SP), observamos en las cajas de dispersión tanto para el grupo control como para el experimental un casi total traslapamiento de las distribuciones (cont. figs. 2 y 3), sobre todo en el grupo experimental, El perfil de medias (fig. 1) por su parte da cuenta de la forma en que las medias para ambos grupos en las dos condiciones antes-después se mantienen casi constantes.

Los relativos cambios observados entre las dos aplicaciones, pretest postest, para ambos grupos resultan no significativos con una $\mathrm{p}>$ 0.10 (tablas 1 y 2).

Con respecto al rasgo Flexibilidad (FX) encontramos cambios no significativos en ambos grupos con una $\mathrm{p}>0.10$ (tablas $1 \mathrm{y}$ 2).

El perfil de medias (fig. 1) nos brinda información del ligero cambio que se observa en el grupo experimental, que como ya se mencionó resultó no significativo, así también nos permite ver el ligero cambio decreciente, aunque no significativo, en el grupo control.

Las cajas de dispersión para el grupo experimental muestran el traslapamiento entre las dos condiciones de aplicación (cont. fig. 2) en donde se observa que la media del postest todavía se encuentra dentro del área de la segunda desviación de la distribución del pretest.

En las cajas de dispersión para el grupo control (cont. fig. 3) observamos como se dio un cambio negativo -aunque como ya se hizo mención es no significativo- percibiendo como el área de la segunda desviación del postest, abarca parte de la primera desviación del pretest.

Por último el rasgo Feminidad (FE) se presenta con unos ligeros cambios en las medias de las aplicaciones pretest-postest en ambos grupos (fig. 1). Sin embargo estos cambios resultan no significativos para ambos grupos al poseer una $\mathrm{p}>0.10$ (tablas 1 y 2).

Las cajas de dispersión para el grupo experimental permiten visualizar la sobreposición (cont. fig. 2) en donde se observa la media del postest dentro del área de la segunda desviación del pretest. Las cajas de dispersión para el grupo control (cont. fig. 3) dan cuenta de una situación similar a la del grupo experimental.

Análisis entre las relaciones secundarias del diseño 
Carvallo Castillo, R. A. (2001). Desarrollo de rasgos asociados a la autoestima a través de la metacognición, en una universidad mexicana. RELIEVE, v. 7, n. 2, p. 135-153.

http://www.uv.es/RELIEVE/v7n2/RELIEVEv7n2_5.htm

E1

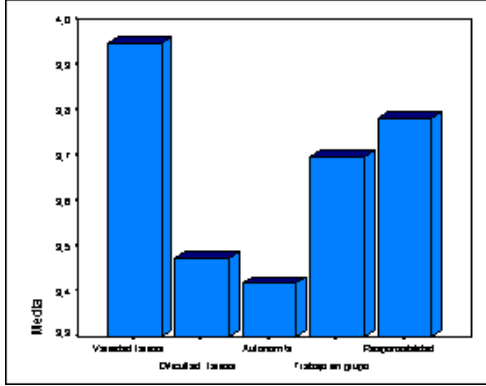

$\mathrm{C} 1$

Este análisis comparativo hace referencia a las diferentes condiciones de medición entre el grupo control y el grupo experimental.

La comparación entre la primera aplicación (pretest) del grupo experimental (E1) y del grupo control (C1) con las pruebas Lambda y Bartlett dan como resultado en sus efectos
E2

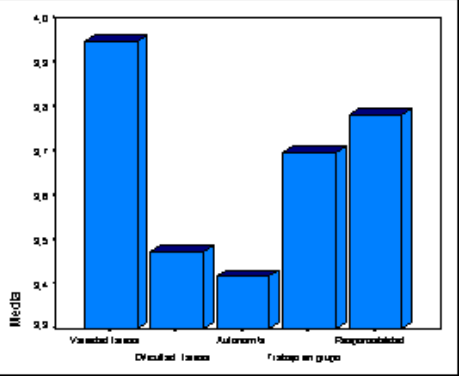

$\mathrm{C} 2$

\begin{tabular}{|l|c|c|c|c|}
\hline \multicolumn{5}{|c|}{ TABLA 3. } \\
\hline & $\begin{array}{c}\text { Pfecto de la media } \\
\text { cuadrada }\end{array}$ & $\begin{array}{c}\text { Error de la media } \\
\text { cuadrada }\end{array}$ & $\mathrm{F}(\mathrm{df1}, 2) 1,50$ & Nivel-p \\
\hline DO & 0.1356 & 88.8261 & 0.001527 & 0.968984 \\
\hline CC & 28.7756 & 161.6133 & 0.178052 & 0.674861 \\
\hline SD & 18.2336 & 62.2815 & 0.292761 & 0.590859 \\
\hline PS & 3.2741 & 128.0741 & 0.025564 & 0.873614 \\
\hline AA & 3.4701 & 73.0641 & 0.047494 & 0.828369 \\
\hline SB & 0.5908 & 122.6928 & 0.004815 & 0.944956 \\
\hline RE & 14.9751 & 84.1170 & 0.178027 & 0.674883 \\
\hline SN & 1.2564 & 167.8933 & 0.007483 & 0.931409 \\
\hline AC & 31.3505 & 87.3618 & 0.358858 & 0.551846 \\
\hline TO & 3.1026 & 99.1733 & 0.031284 & 0.860322 \\
\hline BI & 18.3249 & 87.0073 & 0.210613 & 0.648276 \\
\hline CO & 2.4962 & 182.4516 & 0.013681 & 0.907355 \\
\hline LC & 4.1574 & 162.9122 & 0.025519 & 0.873724 \\
\hline LI & 1.5600 & 71.7888 & 0.021730 & 0.883399 \\
\hline EI & 4.5584 & 144.3304 & 0.031583 & 0.859664 \\
\hline SP & 279.1140 & 82.4593 & 3.384871 & 0.071737 \\
\hline FX & 16.3262 & 103.4900 & 0.157756 & 0.692921 \\
\hline FE & 88.8534 & 70.7414 & 1.256032 & 0.267758 \\
\hline
\end{tabular}

Cont. TABLA 3

Prueba de significancia ( anova, manova) entre los grupos E1-C1.

\begin{tabular}{|l|c|c|}
\hline \multicolumn{1}{|c|}{ Prueba } & Valor & Nivel-p \\
\hline Wilks' Lambda & 0.772628 & \\
Rao R Form 2 (18, 33) & 0.539521 & 0.91633 \\
\hline Pillai-Bartlett Trace & 0.227372 & \\
V $(18,33)$ & 0.539521 & 0.91633 \\
\hline
\end{tabular}


Carvallo Castillo, R. A. (2001). Desarrollo de rasgos asociados a la autoestima a través de la metacognición, en una universidad mexicana. RELIEVE, v. 7, n. 2, p. 135-153.

http://www.uv.es/RELIEVE/v7n2/RELIEVEv7n2_5.htm

El análisis multivariado entre los puntajes de la primera aplicación del grupo experimental (E1) y los de la segunda aplicación del grupo control (C2) da como resultado en sus efectos globales, como era de esperarse, diferencias no significativas con una $\mathrm{p}>$ 0.10. Sin embargo en algunos rasgos indivi- duales tales como Responsabilidad (RE) Comunalidad (CO), Sentido Psicológico (SP) y Feminidad (FE) se observan cambios significativos con una $\mathrm{p}<0.05$, que deberán atribuirse a características propias del grupo control, en cuanto al aspecto escolar (Tabla $4)$..

\begin{tabular}{|l|c|c|c|c|}
\hline \multicolumn{5}{|c|}{ Trueba de significancia (anova manova), entre los grupos E1-C2 } \\
\hline & $\begin{array}{c}\text { Efecto de la } \\
\text { media cuadrada }\end{array}$ & $\begin{array}{c}\text { Error de la media } \\
\text { cuadrada }\end{array}$ & F 1,50 & Nivel-p \\
\hline DO & 19.250 & 81.1365 & 0.237258 & 0.628323 \\
\hline CC & 123.853 & 142.2933 & 0.870403 & 0.355327 \\
\hline SD & 23.128 & 58.8063 & 0.393295 & 0.533426 \\
\hline PS & 148.103 & 90.6533 & 1.633724 & 0.207091 \\
\hline AA & 74.954 & 53.2625 & 1.407257 & 0.241119 \\
\hline SB & 127.442 & 148.1600 & 0.860167 & 0.358148 \\
\hline RE & 313.461 & 71.9442 & 4.357003 & 0.041974 \\
\hline SN & 315.354 & 157.1125 & 2.007186 & 0.162756 \\
\hline AC & 25.227 & 95.8658 & 0.263153 & 0.610221 \\
\hline TO & 250.293 & 82.9941 & 3.015795 & 0.088615 \\
\hline BI & 7.733 & 70.3849 & 0.109872 & 0.741675 \\
\hline CO & 1360.739 & 148.0756 & 9.189484 & 0.003850 \\
\hline LC & 147.065 & 128.4802 & 1.144651 & 0.289809 \\
\hline LI & 3.332 & 85.2672 & 0.039081 & 0.844090 \\
\hline EI & 491.379 & 146.3736 & 3.357021 & 0.072878 \\
\hline SP & 389.396 & 77.3825 & 5.032100 & 0.029343 \\
\hline FX & 98.776 & 123.0660 & 0.802628 & 0.374602 \\
\hline FE & 514.601 & 91.1926 & 5.643015 & 0.021400 \\
\hline
\end{tabular}

\begin{tabular}{|l|c|c|}
\hline \multicolumn{2}{|c|}{ Prueba de significancia (anova manova), entre los grupos E1-C2 } \\
\hline \multicolumn{1}{|c|}{ Prueba } & Valor & Nivel-p \\
\hline Wilks' Lambda & 0.562608 & \\
Rao R Form 2 $(18,33)$ & 1.4253 & 0.184019 \\
\hline Pillai-Bartlett Trace & 0.437392 & \\
V $(18,33)$ & 1.4253 & 0.184019 \\
\hline
\end{tabular}

En la comparación entre el postest del grupo experimental (E2) y el pretest del grupo control (C1) aunque encontramos en el análisis multivariado que las diferencias son nulas con una $p>0.10$, a nivel univariado encontramos diferencias significativas con una $\mathrm{p}<$
0.05 en tres de los principales rasgos asociados a la autoestima, siendo estos, la Autoaceptación (AA), el Sentido de Bienestar (SB) y la Sociabilidad (SD), este último obtuvo una diferencia significativa con una $\mathrm{p}<$ 0.005 (tabla 5) 
Carvallo Castillo, R. A. (2001). Desarrollo de rasgos asociados a la autoestima a través de la metacognición, en una universidad mexicana. RELIEVE, v. 7, n. 2, p. 135-153.

http://www.uv.es/RELIEVE/v7n2/RELIEVEv7n2_5.htm

\begin{tabular}{|l|c|c|c|c|}
\hline \multicolumn{5}{|c|}{ TABLA 5. Prueba de significancia (anova-manova)entre los grupos E2-C1. } \\
\hline & $\begin{array}{c}\text { Efecto de la media } \\
\text { cuadrada }\end{array}$ & $\begin{array}{c}\text { Error de la media } \\
\text { cuadrada }\end{array}$ & F 1,50 & Nivel-p \\
\hline DO & 0.1356 & 102.6261 & 0.001322 & 0.971144 \\
\hline CC & 84.2849 & 149.7481 & 0.562844 & 0.456632 \\
\hline SD & 583.3511 & 65.0726 & 8.964622 & 0.004271 \\
\hline PS & 180.0626 & 137.4341 & 1.310174 & 0.257813 \\
\hline AA & 305.3868 & 72.4507 & 4.215096 & 0.045316 \\
\hline SB & 429.1214 & 103.8187 & 4.133372 & 0.047372 \\
\hline RE & 1.4423 & 56.4400 & 0.025555 & 0.873637 \\
\hline SN & 9.7500 & 159.0000 & 0.061321 & 0.805435 \\
\hline AC & 171.1510 & 71.3781 & 2.397808 & 0.127812 \\
\hline TO & 0.5990 & 82.0415 & 0.007301 & 0.932247 \\
\hline BI & 24.2715 & 65.6296 & 0.369825 & 0.545852 \\
\hline CO & 151.2369 & 168.4368 & 0.897885 & 0.347907 \\
\hline LC & 76.9074 & 141.6722 & 0.542854 & 0.464695 \\
\hline LI & 23.9047 & 76.4481 & 0.312692 & 0.578529 \\
\hline EI & 252.1546 & 118.0904 & 2.135268 & 0.150202 \\
\hline SP & 240.4281 & 81.1926 & 2.961207 & 0.091468 \\
\hline FX & 57.2856 & 62.9389 & 0.910178 & 0.344657 \\
\hline FE & 0.3834 & 53.9369 & 0.007108 & 0.933149 \\
\hline
\end{tabular}

\begin{tabular}{|l|c|c|}
\hline \multicolumn{3}{|c|}{ Cont. TABLA 5. } \\
\hline Prueba de significancia (anova-manova) entre los grupos E2-C1. \\
\hline Wilks' Lambda & Valor & Nivel-p \\
Rao R de 2 (18, 33) & 0.55437 & \\
\hline Pillai-Bartlett Trace & 1.473723 & 0.162986 \\
V (18, 33) & 0.44563 & \\
\hline
\end{tabular}

En el análisis multivariado para las diferencias entre los puntajes del postest tanto del grupo experimental (E2) como del grupo control (C2) encontramos que esta diferencia es significativa con una $\mathrm{p}<0.01$ (tabla 6)

\begin{tabular}{|l|c|c|c|c|}
\hline \multicolumn{5}{|c|}{ TABLA 6. } \\
\hline & $\begin{array}{c}\text { Pfueba de significancia (anova manova), entre los grupos E2-C2 } \\
\text { cuadrada }\end{array}$ & $\begin{array}{c}\text { Error de la media } \\
\text { cuadrada }\end{array}$ & F 1,50 & Nivel-p \\
\hline DO & 19.2503 & 94.9365 & 0.20277 & 0.654441 \\
\hline CC & 11.6695 & 130.428 & 0.089471 & 0.766091 \\
\hline SD & 227.207 & 61.5974 & 3.688586 & 0.060498 \\
\hline PS & 0.3141 & 100.013 & 0.003141 & 0.955532 \\
\hline AA & 48.3706 & 52.6491 & 0.918736 & 0.342419 \\
\hline SB & 103.934 & 129.286 & 0.803912 & 0.374223 \\
\hline RE & 226.082 & 44.2672 & 5.10722 & 0.028215 \\
\hline SN & 248.271 & 148.219 & 1.675024 & 0.201533 \\
\hline AC & 186.566 & 79.8821 & 2.335521 & 0.132755 \\
\hline TO & 336.944 & 65.8623 & 5.115881 & 0.028088 \\
\hline BI & 143.718 & 49.0072 & 2.9326 & 0.093004 \\
\hline CO & 529.479 & 134.061 & 3.949546 & 0.052376 \\
\hline LC & 1.73815 & 107.24 & 0.016208 & 0.899205 \\
\hline LI & 18.6001 & 89.9265 & 0.206837 & 0.651226 \\
\hline
\end{tabular}


Carvallo Castillo, R. A. (2001). Desarrollo de rasgos asociados a la autoestima a través de la metacognición, en una universidad mexicana. RELIEVE, v. 7, n. 2, p. 135-153.

http://www.uv.es/RELIEVE/v7n2/RELIEVEv7n2_5.htm

\begin{tabular}{|l|l|l|l|l|}
\hline EI & 17.2446 & 120.134 & 0.143545 & 0.706386 \\
\hline SP & 343.441 & 76.1158 & 4.512088 & 0.038625 \\
\hline FX & 464.313 & 82.5149 & 5.627014 & 0.021575 \\
\hline FE & 192.593 & 74.3882 & 2.589023 & 0.113903 \\
\hline
\end{tabular}

\begin{tabular}{|l|c|c|}
\hline \multicolumn{3}{|c|}{ Cont. TABLA 6 } \\
\hline Prueba de significancia (anova manova), entre los grupos E2-C2 \\
\hline Prueba & Valor & Nivel- p \\
\hline Wilks' Lambda & 0.404875 & \\
Rao R Form 2 (18, 33) & 2.694818 & 0.006584 \\
\hline Pillai-Bartlett Trace & 0.595125 & \\
V (18,33) & 2.694818 & 0.006584 \\
\hline
\end{tabular}

En el análisis univariado (Tabla 6) observamos diferencia significativa con una $\mathrm{p}<$ 0.05 en dos rasgos asociados de alguna manera a la autoestima y que son Flexibilidad (FX) y Tolerancia (TO) y otros dos con una $\mathrm{p}<0.10$ que son la Sociabilidad (SD), y la Buena Impresión (BI). Existen otros tres rasgos significativos también con $\mathrm{p}<0.05$ pero probablemente mas asociados a los aspectos escolares, estos son Responsabilidad (RE), Sentido Psicológico (SP) y Comunalidad (CO) (tabla 6).

\section{DISCUSIÓN}

En la presente investigación observamos con respecto a los cambios entre el pretest y el postest del grupo experimental (E1, E2) que en el análisis multivariable se produjo una diferencia significativa extrema, lo cual en términos generales implica cambios en la personalidad.

En el análisis por rasgo hallamos que varios de estos asociados con la autoestima, tuvieron cambios significativos relacionados con los dos elementos principales de la autoestima que son eficacia personal y el sentido del mérito personal, con respecto al primer componente (autoeficacia) encontramos los rasgos autocontrol, eficacia intelectual y sociabilidad (y extremadamente cercano, el rasgo presencia social) respecto del segundo componente (sentido de merito) encontramos los rasgos autoaceptación y el sentido de bienestar. Lo anterior nos lleva a concluir que se lograron cambios en el sentido esperado.

El análisis multivariado para los cambios del pretest - postest del grupo control (C1C2) en el total de rasgos proporcionó cambios no significativos. No obstante muestra un cambio significativo particular en el rasgo comunalidad el cual deberá adjudicarse a otras variables no manipuladas en el estudio como por ejemplo los profesores o profesoras de las diferentes materias del curriculum.

El hecho de que no se encontraran diferencias significativas ni de manera global ni por rasgo entre el pretest del grupo experimental (E1) y el pretest del grupo control (C1) nos permite validar el muestreo aleatorio realizado para lo conformación de ambos grupos, y en este caso aseverar con mayor grado de certeza que los cambios encontrados en los rasgos en el postest se deben a variables a los que los alumnos fueron expuestos.

Como se vio en los resultados, entre la primera aplicación del grupo experimental (E1) y la segunda aplicación del grupo control (C2) no existió una diferencia significativa global, lo cual esta en dirección de lo esperado. Sin embargo a nivel de rasgos particulares se encontraron algunos cambios. Uno de ellos ya mencionado arriba para el grupo control fue el del rasgo comunalidad y que como también se hipotetizó podría estar relacionado con variables académicas in- 
Carvallo Castillo, R. A. (2001). Desarrollo de rasgos asociados a la autoestima a través de la metacognición, en una universidad mexicana. RELIEVE, v. 7, n. 2, p. 135-153.

http://www.uv.es/RELIEVE/v7n2/RELIEVEv7n2_5.htm

herentes a ese grupo ya que en este caso también se ve el cambio en otros rasgos asosiados, como en el caso de responsabilidad y el de sentido psicológico, haciendo este último referencia al interés por las necesidades y motivaciones de los demás, lo cual a su vez ajusta (teóricamente) con el rasgo de feminidad que también cambio significativamente, y con el de comunalidad expresado antes.

En cuanto al análisis realizado entre el postest del grupo experimental (E2) y el pretest del grupo control (C1) observamos que de manera global no presento diferencia significativa, aunque estuvo muy cerca, y eso puede deberse a que solo en una pequeña porporción de rasgos -tres de los dieciocho-, se obtuvo un cambio significativo, sin embargo estos tres rasgos están en dirección de lo esperado ya que los tres forman parte de los dos factores involucrados en la autoestima, esto es, la eficacia personal, representada en este caso por el rasgo sociabilidad, el cual requiere confianza en sí mismo y el factor denominado sentido de mérito personal, representado aquí por los rasgos autoaceptación y sentido de bienestar.

El último análisis en este caso y referido a la comparación entre el postest del grupo experimental (E2) y el postest del grupo control (C2) en cuanto al análisis global, es decir el del conjunto de rasgos resulta como es de esperarse, con diferencias significativas entre ambos grupos. Sin embargo adentrándose en el análisis por rasgo significativo, observamos como la diferencia obedece tanto a variables inherentes al grupo experimental como a los factores que influyeron particularmente en el grupo control.

Puede observarse como rasgos que resultaron significativos en la comparación entre el pretest del grupo experimental y el postest del grupo control (E1-C2) resultan también significativos en la comparación presente (E2-C2); estos rasgos son el de responsabilidad y el de sentido psicológico; como ya se comentó anteriormente este cambio podía estar relacionado con variables académicas inherentes a este grupo control ya que varía con el grupo experimental en sus dos exposiciones (pretest-postest).

Una de las variables en que varían significativamente el grupo experimental del control en la condición postest (E2-C2) es la sociabilidad y la flexibilidad, que en su sentido de adaptabilidad están asociados a la autoestima en cuanto a su competencia y seguridad.

Con base a lo arriba expuesto podemos señalar en términos generales y en dirección de la hipótesis alterna -la cual aceptamos-, que los promedios de los puntajes obtenidos por los alumnos en el Inventario Configuración Psicológica Individual en aquellos rasgos que están más relacionados con el desarrollo humano y particularmente con la autoestima son significativamente favorables, por lo tanto podemos concluir que la aplicación de programas con estrategias metacognitivas dirigidas para el desarrollo personal de los jóvenes, produce cambios sustanciales en los rasgos relacionados con el objetivo.

\section{BIBLIOGRAFIA}

Alonso, J (1994). Motivación y aprendizaje en el aula: Como enseñar a pensar. Santillana, S.A.,Madrid.

Alonso, J. (1992). Leer, comprender y pensar: Nuevas estrategias y técnicas de evaluación. C.I.D.E., Madrid.

Anderson, C. A. y cols. (1994). "Atributional style of lonely and depressed people". Journal of Personality and Social Psychology, 45: 127-136.

Arkin, R. y cols. (1988). "Social anxiety, self-presentation and the self-serving bias in causal attributions". Journal of Personality and Social Psychology. 38:23-35.

Ausubel, D. (1980). Psicología de la educación. Un punto de vista cognoscitivo. Trillas, México.

Bandura, A. (1991). Pensamiento y acción. Fundamentos Sociales. Martínez Roca. Barcelona. 
Carvallo Castillo, R. A. (2001). Desarrollo de rasgos asociados a la autoestima a través de la metacognición, en una universidad mexicana. RELIEVE, v. 7, n. 2, p. 135-153.

http://www.uv.es/RELIEVE/v7n2/RELIEVEv7n2_5.htm

Bandura, A. (1989). "Percerved self-efficacy in the exercise of personal agency". The Psychologist. 10: 411-424.

Bednar, R. (1991). "Self-Esteem: Paradoxes and Innovations in Clinical Theory and Practice”. American Psychology Association, Washington.

Belmont, J. M. (1990) "Cognitive strategies an strategie learning”. American Psychologist, 44: 142-148.

Bem, D. (1982). "Self-perception theory", en L. Berkowitz (de.). Advances in experimental social psychology. Academic. Vol. 6. Nueva York.

Branden, N. (1996). Los seis pilares de la autoestima. Paidós, México.

Branden, N. (1994). El poder de la autoestima. Paidós, México.

Bruner, J. y Goodman, C. (1947). "Value as need as organizing factores in perception". Journal of Abnormal and Social Psychology. 43: 23-44.

Coopersmith, S. (1991). The antecedent of self-esteem. Freman. San Francisco.

De Bono, E. (1998). Pensar bien. Selector. México

De Bono, E. (1994). Saber pensar. Ed. Selector. México.

De Sánchez, M.A. (1996). Desarrollo de habilidades del pensamiento: procesos_básicos del pensamiento. Trillas, México.

Dendaluce, I. (1988). Aspectos metodológicos de la investigación educativa, Narcea, España.

Echebarría, A. (Ed.), (1991). Psicología social sociocognitiva. Desclée de Bowner, Bilbao.

Echebarría, A. y Villarreal, M. (1991). "La percepción Social”, en Echebarría ed. Psicología Social Sociocognitiva. DDB, Bilbao.

Eiser, J. R. (1990). Psicología Social: actitudes, cogniciones y conducta social. Pirámide, Madrid.

Erser, J. R. (1983). "Attribution theory and social cognition", en J. Jaspars y cols. (eds.), Attribution theory and research: conceptual, developmental and social dimension.. Academic, Londres.
Evans, P. (1992). Motivación. Cía. Ed. Continental, México.

Fiske, S. y Taylor, S. (1994). Social cognition. McGraw-Hill, 2th Ed., Nueva York.

Flavell, J. (1985). "Speculations about the nature and development of metacognition", en F. Weinert y R. Kluwe (eds.) Metacognition, motivation and understanding. LEA, Hillsdale.

Goleman, D. (1998). Inteligencia emocional. Javier Vergara Editor, Buenos Aires.

Gough, H.G.(1990). Configuración Psicológica Individual. Editorial Manual Moderno, México

Higgins, E. T. y Bargh, J. A. (1989). "Social cognition a social perception". Annual Review of Psychology, 38: 369-425.

León, J. y Gómez, T. (1998). "Percepción Social”, en León, J. y otros, Psicología Social. McGraw-Hill. España.

León R., J. (1998). “Control psicológico”, en León, J. y otros, (1998), Psicología_Social. McGraw-Hill, España.

León, J. y Gómez, T. (1998). “Atribución Causal”, en León, J. y otros Psicología Social. McGraw-Hill, España.

León, J.; Medina, S.; Barriga S. y Cantero, F. (1998). "Bases Sociales de la emoción”, en León, J. y otros, Psicología Social.McGrawHill, España.

Loscertales, F. (1998). "Construcción Social de la identidad personal”,en León, J. y otros, Psicología Social. McGraw-Hill.

Markus, H. y Wurf, E. (1990). "The dynamic self-concept: A social psychological perspective". Annual Review of Psychology Bulletin, 8: 189-194.

Ornstein, P. (1994). "Knowledge and strategies”, en W. Schneider y F. Weinert (eds.) Interactions among aptitudes, strategies and knowledge in cognitive performance. Springer, Nueva York.

Ross, L. y Anderson, C. (1992). "Shortcomings in the attribution procesess: on the origins and maintenance of erroneous social assessments", en D. Kahneman y cols. (eds.), Judgment under uncertainty: Heuristics and biases. Cambridge University Press. Nueva York. 
Carvallo Castillo, R. A. (2001). Desarrollo de rasgos asociados a la autoestima a través de la metacognición, en una universidad mexicana. RELIEVE, v. 7, n. 2, p. 135-153.

http://www.uv.es/RELIEVE/v7n2/RELIEVEv7n2_5.htm

Schachter, S y Singer, J. (1962). "Cognitive, social and physiological determinants of emotional state". Psychological Review, 69: 379-399.

Schlenker, B. y Wigold, M. (1992). "Interpersonal processes involving impression regulation and management”. Annual Review of Psychology, 43.

Schunck D. (1990). "Social cognitive theory and self-regulated learning”, en B. Zimmerman y D. Schunck (eds.), Self regulated learning and academic achievement. Springer, Nueva York.

Schwartz, G. Shapiro, D. Y Davidson R. (1986). Consciousness and self-regulation. Vol.4, Plenum, Nueva York.

Steele, C. (1995). "The psychology of selfaffirmation: sustaining the integrity of the self". en Berkowitz (de.), Advances in experimental social psychology, Vol. 21. Academic Press. Orlando, FL.

Taylor, N. (1990). "Metacognitive ability: a curriculum priority". Reading Psychology an International Quarterly. 4: 269-278.

Tice, D. (1991). "Esteem protection or enhancement? Self-handicapping motives and attributions differ by trait self-esteem". Journal of Personality and Social Psychology, 60: 711-725.

Wong, B. (1994). "The relevance of metacognition to learning disabilities”, en B. Wong (ed.), Learning about learning disabilities. Academic, San Diego.

Wylie, R. C. (1992). "The self-concept". Vol. II, Theory and research on selected topics. University of Nebraska, Press. Lincoln.

\section{ANEXO 1}

Programa de estrategias metacognitivas para el desarrollo del sí mismo: autoestima y factores asociados Presentación General:

Módulo 1. Búsqueda de información adecuada y construcción del significado: El lector es como un investigador privado

Unidad 1: Busca información adecuada y construye el significado: El lector es como un investigador privado.

Unidad 2: Encuentra el significado: El lector es como un investigador privado

Módulo 2. Las impresiones del significado del texto y su aplicación en el desarrollo personal: La lectura como guía de tu vida

Unidad 1: Implicación del significado en la vida cotidiana: La lectura como guía de tu vida.

Unidad 2: Despertar de impresiones: La lectura como guía de tu vida

Módulo 3. Soy competente para vivir y digno de ser feliz: El camino hacia la autoestima

Unidad 1: Entendiendo mi autoestima: El primer paso a mi plenitud

Unidad 2: Conociendo mi sí-mismo que llevo dentro: Mi autoconcepto

$\underline{\text { Unidad 3: }}$ Integrando mi pasado y mi presente: mi sí-mismo niño, mi sí-mismo adolescente y mi sí-mismo adulto hermanados.

Unidad 4:Saber qué hacer y tener conciencia sobre ello: Vivir conscientemente.

Módulo 4. Confianza en la eficacia de nuestra mente: Eliminando mis obstáculos y pensando conscientemente me dirijo hacia mi plenitud

Unidad 1: En este momento me percibo así: Para cambiar hay que aceptarse

Unidad 2: Conspiración de autoculpabilidad: Eliminemos este sentimiento

Unidad 3: Responsabilidad ante mí y respeto mis valores para ser auténtico

Unidad 4: Estrategias metacognitivas generales a considerar en el desarrollo de la autoestima.

MÓDULO 5. Relaciones interpersonales efectivas: Cómo comunicarme sin daño humano

Unidad 1: Las cosas mal dichas afectan nuestras relaciones personales: No decir cosas destructivas

Unidad 2: Aprendiendo a ser positivo en mi comunicación: Cómo decir las cosas constructivamente.

Unidad 3: Continuando con mi comunicación positiva 
Carvallo Castillo, R. A. (2001). Desarrollo de rasgos asociados a la autoestima a través de la metacognición, en una universidad mexicana. RELIEVE, v. 7, n. 2, p. 135-153.

http://www.uv.es/RELIEVE/v7n2/RELIEVEv7n2_5.htm

\section{ARTICLE RECORD / FICHA DEL ARTÍCULO}

\begin{tabular}{|c|c|}
\hline $\begin{array}{l}\text { Reference / } \\
\text { Referencia }\end{array}$ & $\begin{array}{l}\text { Carvallo Castillo, R. a. (2001). Desarrollo de rasgos asociados a la autoestima a través de la metacogni- } \\
\text { ción, en una universidad mexicana. RELIEVE, vol. 7, n. 2. Consultado en } \\
\text { www.uv.es/RELIEVE/v7n2/RELIEVEv7n2_5.htm en (poner fecha). }\end{array}$ \\
\hline $\begin{array}{l}\text { Title / } \\
\text { Título }\end{array}$ & $\begin{array}{l}\text { Desarrollo de rasgos asociados a la autoestima a través de la metacognición en una universidad mexi- } \\
\text { cana [Development of traits related to self-esteem through metacognition in a Mexican university ] }\end{array}$ \\
\hline $\begin{array}{l}\text { Authors / } \\
\text { Autores }\end{array}$ & Carvallo Castillo, Raúl Augusto \\
\hline $\begin{array}{l}\text { Review / } \\
\text { Revista }\end{array}$ & Revista ELectrónica de Investigación y EValuación Educativa (RELIEVE), v. 7, n.2 \\
\hline ISSN & $1134-4032$ \\
\hline $\begin{array}{l}\text { Abstract / } \\
\text { Resumen }\end{array}$ & $\begin{array}{l}\text { We show the effect of the practice of metacognitive strategies for the change in affective-emotive fac- } \\
\text { tors. We present an empirical study on Mexican university students } \\
\text { Se pretende poner en evidencia el efecto que pueden ejercer las estrategias metacognitiva para el cambio } \\
\text { en factores afectivos-emotivos usando una muestra de estudiantes universitarios mexicanos. }\end{array}$ \\
\hline $\begin{array}{l}\text { Keywords / } \\
\text { Descriptores }\end{array}$ & $\begin{array}{l}\text { Self-esteem, metacognition, motivation, university } \\
\text { Autoestima, metacognicion, motivación, universidad }\end{array}$ \\
\hline $\begin{array}{l}\text { Institution / } \\
\text { Institución }\end{array}$ & Universidad Veracruzana (México) \\
\hline $\begin{array}{l}\text { Publication } \\
\text { site / } \\
\text { Dirección }\end{array}$ & http://www.uv.es/RELIEVE \\
\hline $\begin{array}{l}\text { Language / } \\
\text { Idioma }\end{array}$ & Spanish (Title, abstract and keywords in English ) \\
\hline
\end{tabular}

Revista ELectrónica de Investigación y EValuación Educativa (RELIEVE)

[ ISSN: 1134-4032 ]

(C) Copyright, RELIEVE. Reproduction and distribution of this articles it is authorized if the content is no modified and their origin is indicated (RELIEVE Journal, volume, number and electronic address of the document).

(C) Copyright, RELIEVE. Se autoriza la reproducción y distribución de este artículo siempre que no se modifique el contenido y se indique su origen (RELIEVE, volumen, número y dirección electrónica del documento). 